\title{
INTERSECTIONS OF MAXIMAL STARSHAPED SETS
}

\author{
W. R. HARE, JR. AND JOHN W. KENELLY
}

0 . Introduction. In Valentine $[1$, p. 183] the problem of characterizing starshaped sets in terms of maximal convex sets was posed. One published solution says that the convex kernel of a set is the intersection of all the maximal convex subsets of the set $[2$, p. 280]. In this paper we investigate the analogous problem of describing the intersection of all maximal starshaped subsets of a set. A maximal starshaped subset $X$ of a set $Y$ is a starshaped subset of $Y$ which is not properly contained in any other starshaped subset of $Y$. Since the property of being starshaped is not an intersectional property, it seems unlikely that the intersection of maximal starshaped subsets of a given set would be starshaped. Indeed, the following example shows the situation to be even more complex than merely absence of the intersectional property.

Let $T_{n}=\{(x, y) \mid n-1 \leqq y \leqq n, n-x \leqq y\}$, and $S_{n}=\cup_{i=1}^{n} T_{i}$; then $S_{n}$ is starshaped with convex kernel, $\operatorname{ck}\left(S_{n}\right)$, equal to $K_{n}=\{(x, y) \mid 0$ $\leqq y \leqq 1, n-x \leqq y\}$. If $S=\cup_{n=1}^{\infty} S_{n}$, then $\operatorname{ck}(S) \subset \cup_{n=1}^{\infty} \operatorname{ck}\left(S_{n}\right)=\varnothing$. Thus $S$ is not starshaped even though it is the union of an ascending chain of starshaped sets. Furthermore, $S$ has no maximal starshaped subsets. If $M \subset S$ were a maximal starshaped subset, then there would be at least one point $(x, y) \in \operatorname{ck}(M)$. In fact $M$ would be precisely the set of points that $(x, y)$ sees via $S$. However, the point $(x+1, y)$ sees every point which $(x, y)$ does, and more. Thus $M$ is not maximal.

In contrast with the preceding example, it is shown in $\$ 1$ that compact subsets of Euclidean space, $E^{n}$, have maximal starshaped subsets. In $\S 2$, it is shown that the intersection of the maximal starshaped subsets in a suitably restricted setting is starshaped.

1. Existence of maximal starshaped sets. Let $S$ be a compact set in $E^{n}$ and let $\mathcal{F}$ denote the family of all classes $C$ of maximal convex subsets of $S$ for which $\cap C \neq \varnothing$. Observe that a maximal convex subset of $S$ is compact. We note two properties of $\mathcal{F}$. First, if $D$ is a finite subclass of some $C \in \mathcal{F}$ then $\varnothing \neq \cap C \subset \cap D$, so $D \in \mathcal{F}$. Also, if $C$ is a class of sets such that each finite subclass is in $\mathcal{F}$, then $C \in \mathcal{F}$ by compactness and the definition of $\mathscr{F}$. Thus $\mathscr{F}$ is a family of finite character.

THEOREM 1.1. There exists a maximal starshaped subset $T$ of any compact set $S$ in $E^{n}$ and every maximal starshaped subset is closed.

Presented to the Society, August 31, 1967 under the title The intersection of maximal starlike sets; received by the editors July $27,1967$. 
Proof. By the preceding, Tukey's Lemma gives a maximal class $C$ of maximal convex subsets of $S$ for which $\cap C \neq \varnothing$. Let $T=\cup C$; by using the fact that a starshaped set is the union of its maximal convex subsets, we see that $T$ is indeed a maximal starshaped subset of $S$. Noting that the closure, $T^{-}$, is starshaped and $T^{-} \subset S$, we see that $T=T^{-}$. That is, $T$ is closed.

CoROllary 1.2. If $S \subset E^{n}$ is compact and $B$ is any starshaped subset of $S$, then there exists a maximal starshaped subset $T \subset S$ such that $B \subset T$.

Proof. Express $B$ as the union of its maximal convex subsets; then let $T=\cup C$, where $C$ is one maximal class of maximal convex subsets of $S$ with $\cap C \neq \varnothing$, at least one member containing each one of the maximal convex subsets of $B$.

2. Intersections of maximal starshaped sets in the plane. Hereafter $S$ is always taken to be a compact simply connected set in the plane. Likewise $S_{\alpha}, \alpha$ in an index set $I$, will represent a maximal starshaped subset of $S$; and $A$ is taken to be the intersection of all the maximal starshaped subsets of $S$, i.e. $A=\bigcap_{\alpha \in I} S_{\alpha}$. We note that $A$, perhaps empty, is closed and thus compact.

Particular notations are as follows: $p q$ denotes the closed segment established by the points $p$ and $q ; \Delta p q r$ denotes the convex hull of the three points, $p, q$, and $r ; L(p, q)$ is the line established by the points $p$ and $q$; and ${ }_{k} C_{p q}$ denotes the cone opposite $p$ and $q$ with vertex $k$, i.e. ${ }_{k} C_{p q}=\{x \mid x=\lambda p+\mu q+\nu k, \lambda+\mu+\nu=1, \lambda \leqq 0, \mu \leqq 0\}$.

Lemma 2.1. If $p, q \in A$, then $p q \subset A$ if and only if $p q \subset S$.

Proof. The "only if" part is immediate. If $p q \subset S$ and $k \in \operatorname{ck}\left(S_{\alpha}\right)$, then we have $p q \cup q k \cup k p \subset S$. So $\Delta p q k \subset S$. This means that $k$ sees all of $\Delta p q k$, so $S_{\alpha} \cup \Delta p q k$ is a starshaped subset of $S$ having $k$ in its kernel. Consequently we have $p q \subset S_{\alpha}$ by the maximality of $S_{\alpha}$. That is $p q \subset A$.

Observe that simple connectedness and a standard sequence argument gives the following. If $p, q \in A$ and $p q \llbracket A$, then the set of points, $B$, that see $p$ and $q$ via $S$ is a compact set contained in one of the open half planes of $L(p, q)$.

LeMma 2.2. The set $B$, as given above, contains a unique element which is closest to $L(p, q)$.

Proor. Since the distance from points of $B$ to $L(p, q)$ is a positive continuous function defined on a compact set, we observe that a closest point exists. Distinct closest points $x$ and $y$ in $B$ establish 
$L(x, y)$ parallel to $L(p, q)$. Adjusted notation, if necessary, gives $x q \cap y p$ to be a point of $B$ closer than the minimum distance.

Lemma 2.3. Every pair of points of $A$ can be joined in $A$ by a polygonal path of no more than two edges.

Proof. If $p, q \in A$ and $p q \nsubseteq A$, let $m$ be the unique point of Lemma 2.2. If $k \in \operatorname{ck}\left(S_{\alpha}\right)$, we have $k \in{ }_{m} C_{p q}$. But simple connectedness of $S$ gives the quadrilateral $k p m q$ and its interior to be a subset of $S$. Now $k$ sees $m$, so $p m \cup m q \subset S_{\alpha}$. Since $\alpha$ was arbitrary, it follows that $p m \cup m q \subset A$.

THEOREM 2.4. The intersection of the maximal starshaped subsets of a compact, simply connected set in $E_{2}$ is starshaped or empty.

Proof. Let $p, q, r$ be three points of $A$ such that no point of $A$ sees all three points via $A$. Otherwise Krasnoselskii's Theorem says that $A$ is starshaped [1].

For the first case assume that $p, q$, and $r$ are collinear with $q$ between $p$ and $r$.

By our initial assumption $\operatorname{pr} \llbracket A$; suppose $q r \nsubseteq A$ and $p q \subset A$. Then Lemma 2.2 establishes a point $m$ closest to $L(q, r)$ and Theorem 2.3 yields $q m \cup m r \subset A$. As before $\operatorname{ck}\left(S_{\alpha}\right) \subset_{m} C_{q r}$ for any $\alpha$. If $k \in \operatorname{ck}\left(S_{\alpha}\right)$, we have $p k \cup k q \cup r k \subset S$. Simple connectedness gives $p m \subset S$. Thus, Lemma 2.1 says $p m \subset A$ and the resulting contradiction- $p m \cup m q$ $\cup m r \subset A$-assures that $p q \nsubseteq A$. Similarly $q r \nsubseteq A$.

Let us now assume that none of the segments between $p, q$, and $r$ is contained in $A$. Again Lemma 2.2 establishes a point $m$ closest to $L(p, r)$ for the points $p$ and $r$ with $p m \cup m r \subset A$. Select $k \in \operatorname{ck}\left(S_{\alpha}\right)$ and note that $k \in{ }_{m} C_{p r}$. If $m \in k q$, we have a contradiction, so assume that $k q \cap(r m \cup p m)$ is a point distinct from $m$. Without loss of generality let the point of intersection be on $r m$. Now apply Lemma 2.2 to establish a point $n$ closest to $L(p, q)$. The point $n$ must be such that $k \in{ }_{n} C_{p q}$, i.e. $n \in \Delta p k q$. Extend $q m$ to intersect $p k$ in a point $j$. If $n \in \Delta p q j$, we observe that $m q \subset A$, a contradiction. Otherwise, either $n r \subset A$ or $n q$ extended to intersect $m r$ yields a point of $A$ that sees $p, q$ and $r$ via $A$.

We now observe that all the above excludes the possibility of $p, q$ and $r$ being collinear.

Since $p, q$ and $r$ are not collinear, we employ them as a barycentric basis to describe regions of the plane. For example, a $(+,-, 0)$ point $k$ is such that $k=\alpha p+\beta q+\gamma r$ with $\alpha+\beta+\gamma=1$ and $\alpha>0$, $\beta<0, \gamma=0$. 
Suppose $k \in \operatorname{ck}\left(S_{\alpha}\right)$; two cases are trivial-namely $(0,0,+)$ and $(0,+,+)$. Particular permutations of these sign symbols are assumed without loss of generality. The three cases $(0,-,+),(+,-,+)$ and $(-,+,-)$ are disposed of simultaneously (i) with a proof identical in wording to the paragraph that dispenses with $p, q$ and $r$ being collinear and none of their segments in $A$, and (ii) with minor modifications for the cases in which one of the segments $p q, q r, p r$ is in $A$.

The final possibility is for $k \in \operatorname{ck}\left(S_{\alpha}\right)$ to be a $(+,+,+)$-point. Here Lemma 2.2 and Theorem 2.3 assure us that $k p \cup k q \cup k r$ is contained in a "three-pointed star region" all of whose edges are segments of $A$. Simple connectedness and Lemma 2.1 ensure that this region (and, in particular $k p \cup k q \cup k r$ ) is a subset of $A$.

\section{REFERENCES}

1. F. A. Valentine, Convex sets, McGraw-Hill, New York, 1964.

2. F. A. Toranzos, Radial functions of convex and starshaped bodies, Amer. Math. Monthly 74 (1967), 278-280.

Clemson University 